\begin{tabular}{|c|c|c|c|}
\hline \multirow{2}{*}{$\begin{array}{r}\text { Case Reports in } \\
\text { Gastroenterology }\end{array}$} & \multicolumn{2}{|c|}{ Case Rep Gastroenterol 2016;10:633-639 } & \multirow[b]{2}{*}{$\begin{array}{l}\text { Karger } \\
\text { Openaccess }\end{array}$} \\
\hline & $\begin{array}{l}\text { DOI: } 10.1159 / 000452198 \\
\text { Publisned online: October 28, } 2016\end{array}$ & $\begin{array}{l}\text { (c) } 2016 \text { The Author(s) } \\
\text { Published by S. Karger AG, Basel } \\
\text { www.karger.com/crg }\end{array}$ & \\
\hline & $\begin{array}{l}\text { This article is licensed under the } \\
\text { International License (CC BY-NC) } \\
\text { Usage and distribution for commerc }\end{array}$ & $\begin{array}{l}\text { mons Attribution-NonCommercial } 4 . \\
\text { ger.com/Services/OpenAccessLicense } \\
\text { quires written permission. }\end{array}$ & \\
\hline
\end{tabular}

Single Case

\title{
A Rare Cause of Pulmonary Nodules
}

\author{
Michael Tsuyoshi Chew ${ }^{\mathrm{a}}$ Eric Chak $^{\mathrm{b}}$ Karen Matsukuma $^{\mathrm{c}}$ \\ ${ }^{a}$ Department of Internal Medicine, University of California at Davis, Sacramento, CA, USA; \\ ${ }^{b}$ Department of Gastroenterology, University of California at Davis, Sacramento, CA, USA; \\ ${ }^{\mathrm{C}}$ Department of Pathology and Laboratory Medicine, University of California at Davis, \\ Sacramento, CA, USA
}

\section{Keywords}

Pulmonary nodules $\cdot$ Crohn's disease $\cdot$ Extraintestinal manifestations

\begin{abstract}
Crohn's disease is a chronic, idiopathic autoimmune disorder that primarily targets the gastrointestinal (GI) system. It is characterized by transmural inflammation of the GI tract that can occur anywhere from the mouth to the anus. Not infrequently, the disease may also have extraintestinal manifestations (EIMs) that can affect almost any organ system. It is estimated that EIMs affect up to $36 \%$ of patients with Crohn's disease, but the incidence and prevalence of pulmonary involvement are variable in the literature and may be as low as $0.4 \%$. There are few case reports documenting pulmonary manifestations, as they are often overlooked, especially if respiratory symptoms are present before the diagnosis of GI manifestations, as in the present case. A 44-year-old otherwise healthy woman presented with nonspecific respiratory complaints, recurrent pneumonias, and multiple computed tomography images showing diffuse, migratory, nodular, and consolidative parenchymal lung disease, with a largely unremarkable infectious and rheumatologic evaluation. Lung biopsy revealed necrotizing and nonnecrotizing granulomas, raising concern for sarcoidosis. Subsequent imaging revealed an incidental mass in the cecum. Biopsy of the cecum lesion revealed acute cryptitis, crypt abscess, and a single poorly formed granuloma, suggesting the possibility of Crohn's disease. In this report, we present a patient whose pulmonary manifestations ultimately led to the diagnosis of Crohn's disease.




\section{Introduction}

Crohn's disease is a systemic inflammatory disorder that primarily targets the gastrointestinal system, but infrequently the disease may also have extraintestinal manifestations (EIMs) that can affect almost any organ system. EIMs may vary in severity and can be more debilitating than the underlying Crohn's disease. Certain EIMs may parallel Crohn's disease activity, while others can have an independent course. Because EIMs can involve almost any organ system, the clinical presentation is diverse. Well-characterized manifestations include ocular involvement with uveitis and episcleritis, cutaneous manifestations with erythema nodosum and pyoderma gangrenosum, musculoskeletal involvement with reactive arthritis and spondyloarthritis, and hepatobiliary disease [1]. Pulmonary manifestations are less well recognized but can involve airways as well as parenchyma. Because pulmonary involvement is seldom seen with Crohn's disease, making the diagnosis can present a challenge. Moreover, the correlation may be overlooked when the pulmonary disease has an independent course from the gastrointestinal disease, especially when different medical specialists evaluate the patient. We present a case summary of a patient with what we later suspected was pulmonary EIM prior to the diagnosis of Crohn's disease, which is not well defined in the current literature.

\section{Case Report}

A 44-year-old woman was initially referred to the pulmonary clinic in 2012 for recurrent pneumonias, persistent shortness of breath, and chest pain. She said that her clinical course began when she was diagnosed with adult-onset asthma in 2007. Her symptoms were relatively mild until 2011, when she was diagnosed with pneumonia, for which she was treated with antibiotics and short courses of prednisone. Despite treatment, she never felt she fully recovered to her usual state of health. She continued to have episodes of dyspnea on exertion and pleuritic chest pain, complicated by several episodes of pneumonia over the next year and a half.

Physical exam was unremarkable as she was young and appeared to be well. Sclera and conjunctiva were normal, but nasopharyngeal mucosa was slightly erythematous. Cardiac exam was normal and lungs were clear bilaterally. Her abdomen was soft and nontender, and there was no skin or joint involvement.

Initial workup revealed normocytic anemia (hemoglobin, $11.4 \mathrm{gm} / \mathrm{dL}$ ) with peripheral eosinophilia $\left(1.3 \mathrm{k} / \mathrm{mm}^{3}\right)$. She had mixed obstructive and restrictive pattern, with reduced diffusing capacity of the lung for carbon monoxide (DLCO) on pulmonary function testing and multiple computed tomography exams with diffuse, migratory, nodular, and consolidative parenchymal lung disease (Fig. 1a, b). Elevated inflammatory markers corresponded with active symptoms of dyspnea on exertion and pleuritic chest pain (erythrocyte sedimentation rate, $32 \mathrm{~mm} / \mathrm{h}$; C-reactive protein, $2.3 \mathrm{mg} / \mathrm{dL}$ ). Infectious workup was positive for Aspergillus precipitins but negative for coccidioidomycosis, histoplasmosis, Strongyloides, QuantiFERON assay, and HIV screen. There was no evidence of infection or eosinophilia on bronchoscopy. Extensive serologic evaluation revealed weakly positive antinuclear antibodies at 1:80 and IgE in the 200s but was otherwise negative. A PET scan was completed to better characterize the pulmonary lesions, which showed bilateral pleural and parenchymal nodules with the largest in the left lower lobe (Fig. 1c). The PET scan incidentally revealed a mural mass arising from the cecum (Fig. 1d). Computed tomography-guided biopsy of the 


\section{Case Reports in \\ Gastroenterology}

Case Rep Gastroenterol 2016;10:633-639

DOI: $10.1159 / 000452198$

(c) 2016 The Author(s). Published by S. Karger AG, Basel www.karger.com/crg

Chew et al.: A Rare Cause of Pulmonary Nodules

left lower lobe showed predominant necrosis with scant surrounding granulation tissue, negative for acid fast bacilli and without organisms. Due to nonspecific findings, in 2013, she eventually underwent video-assisted thoracoscopic surgery lung biopsy of the right upper, middle, and lower lobes showing a mix of necrotizing and nonnecrotizing granulomas, neutrophilic inflammation, and scattered eosinophils (Fig. 2a-c). The biopsy findings suggested that the patient had chronic, idiopathic granulomatous lung disease, and the decision was made to start the patient on a moderate dose of prednisone and taper her dose over 6 months. Even as her symptoms subsided, she could not be tapered off prednisone. Her symptoms recurred with discontinuation, and she complained of persistent pleuritic chest pain, dyspnea on exertion, headache, fatigue, and diffuse arthralgias.

Our medical team was confounded by her symptomatology in spite of treatment. Upon further review of her past workup, there was evidence of a mural mass in the cecum on PET scan that had not been pursued previously because she had no significant gastrointestinal symptoms. She was referred for colonoscopy in September 2014 with endoscopic findings of isolated nodular and friable cecum (online suppl. Fig., www. karger.com/doi/10.1159/000452198) and patchy erythema in the rectum. The cecal biopsy revealed acute cryptitis, crypt abscess, and a single poorly formed granuloma, thus raising the differential diagnosis of Crohn's disease (Fig. 2d-f). She was started on infliximab and 6-mercaptopurine with significant improvement.

\section{Discussion}

Crohn's disease is a chronic, idiopathic autoimmune inflammatory disorder characterized by transmural inflammation of the gastrointestinal tract that can occur anywhere from the mouth to the anus. It is estimated that EIM affects $21-36 \%$ of patients with Crohn's disease, but the incidence and prevalence of pulmonary involvement varies in the literature [1]. Pulmonary manifestations of Crohn's disease were first described in the late 1960s, but it was not until the mid-1970s when it was widely accepted in the literature as EIM of inflammatory bowel disease (IBD) [2-5]. Pulmonary involvement is not commonly associated with Crohn's disease and often overlooked, especially if respiratory symptoms are present before the diagnosis of gastrointestinal manifestations, as in the present case. It is suggested that ongoing bowel inflammation is not required in order to have respiratory symptoms, as there is embryologic overlap between the respiratory and colonic epithelium [6]. The lungs and the gastrointestinal tract share similar submucosal lymphoid tissue that is crucial in host defense as well as in the development of inflammatory dysregulation $[3,6,7]$.

Most cases of pulmonary EIM occur years after the diagnosis of IBD [8]. However, the diagnosis was delayed in this case as the pulmonary symptoms preceded the IBD diagnosis. To the best of our knowledge, this is one of the few case presentations in an adult with pulmonary EIM before the diagnosis of IBD [6, 8, 9]. Serological testing was negative for IBD and only confirmed on cecal biopsy. Crohn's disease and sarcoidosis are both diseases that result in noncaseating granulomas. Key differentiating points in this case were the peripheral eosinophilia, which is not typically seen in sarcoidosis, and the normal serum angiotensinconverting enzyme levels, with normal serum calcium and 24-h urine collection of calcium [10]. Serum angiotensin-converting enzyme levels are consistently low to normal in Crohn's disease, especially during periods of active inflammation [9]. The patient had normocytic anemia with iron studies suggestive of anemia of chronic inflammation with iron deficiency (ferritin, $8 \mathrm{ng} / \mathrm{mL}$; iron percent saturation, 11\%; and red blood cell distribution width, 17 
units), which is suggestive of Crohn's disease, but not typically seen in sarcoidosis [10]. There was low suspicion for infectious etiologies given the negative serologies, cultures, and special stains performed on the open lung biopsy. The lung pathology did not fit a specific noninfectious granulomatous lung disease. Granulomatosis with polyangiitis was briefly considered given the granulomatous inflammation, radiographic distribution, history of asthma, and peripheral eosinophilia. However, in this case, the absence of necrotizing vasculitis and negative anti-PR3 serology did not support the diagnosis of granulomatosis with polyangiitis.

Pulmonary EIM in Crohn's disease remains underrecognized largely due to the heterogeneous and wide spectrum of involvement. In this case, pulmonary manifestations preceded the detection and, ultimately, led to the diagnosis of Crohn's disease. As highlighted in this case, it is important to complete an extensive infectious, rheumatologic, and pathologic evaluation. The break in this case came when our team decided to further investigate the mural mass in the patient's cecum by obtaining an endoscopic evaluation with biopsies. It was through the workup of her colonic findings that the pulmonary disorder was discovered to be a manifestation of Crohn's disease.

Pulmonary function tests have been studied in patients with IBD, but results have been subclinical and often mixed, which is thought to be due to inadequate power. However, compared to normal subjects, those with IBD consistently had decreased $\mathrm{D}_{\mathrm{LCO}}$, and it may be significantly lower during active disease [4, 6]. Additionally, corticosteroids are considered the mainstay of therapy in treating pulmonary EIM in Crohn's disease [11]. However, up to one-third of patients fail to respond to treatment or cannot taper off, as seen in this patient. She was started on infliximab and 6-mercaptopurine with significant improvement in her pulmonary symptoms. After 1 year of dual therapy, the patient was transitioned to single therapy with infliximab and continues to do well.

The take-away lesson of this case is that diseases do not always present in textbook fashion. In particular, EIMs of Crohn's disease may baffle even the most astute clinician, especially when the extraintestinal involvement precedes any significant inflammatory signs and symptoms of gastrointestinal disease. After exhaustive evaluation of her pulmonary symptomatology, it was the patient's PET scan, and a little serendipity, that led us to further evaluate her for IBD. With the diagnosis of Crohn's disease, we made the association that her pulmonary disease was the manifestation of IBD.

\section{Acknowledgements}

This unique case report would not have been identified without the work of Dr. Jason Adams from the Department of Pulmonary and Critical Care Medicine and Dr. Jesse Stondell from the Department of Gastroenterology, University of California at Davis, Sacramento, CA, USA.

\section{Statement of Ethics}

The patient provided written consent prior to publication of this case report. 
Chew et al:: A Rare Cause of Pulmonary Nodules

\section{Disclosure Statement}

The authors of this case report have no conflicts of interest.

\section{References}

Juillerat P, et al: Extraintestinal manifestations of Crohn's disease. Digestion 2007;76:141-148.

Kraft SC, et al: Unexplained bronchopulmonary disease with inflammatory bowel disease. Arch Intern

Med 1976;136:454-459.

3 Lu DG, et al: Pulmonary manifestations of Crohn's disease. World J Gastroenterol 2014;20:133-141.

-4 Songur N, et al: Pulmonary function tests and high-resolution CT in the detection of pulmonary involvement in inflammatory bowel disease. J Clin Gastroenterol 2003;37:292-298.

-5 Turner-Warwick M: Fibrosing alveolitis and chronic liver disease. Q J Med 1968;37:133-149.

-6 Black H, Mendoza M, Murin S: Thoracic manifestations of inflammatory bowel disease. Chest 2007;131:524-532.

7 Bernstein CN, Wajda A, Blanchard JF: The clustering of other chronic inflammatory diseases in inflammatory bowel disease: a population-based study. Gastroenterology 2005;129:827-836.

8 Casey MB, et al: Noninfectious lung pathology in patients with Crohn's disease. Am J Surg Pathol 2003;27:213-219.

99 Levenbrown Y, et al: Granulomatous lung disease as the initial presentation of Crohn disease. J Pediatr Gastroenterol Nutr 2009;48:487-490.

10 Beniwal RS, Cummings OW, Cho WK: Symptomatic gastrointestinal sarcoidosis: case report and review of the literature. Dig Dis Sci 2003;48:174-178.

11 Hayek AJ, Pfanner TP, White HD: Inflammatory bowel disease of the lung: the role of infliximab? Respir Med Case Rep 2015;15:85-88. 

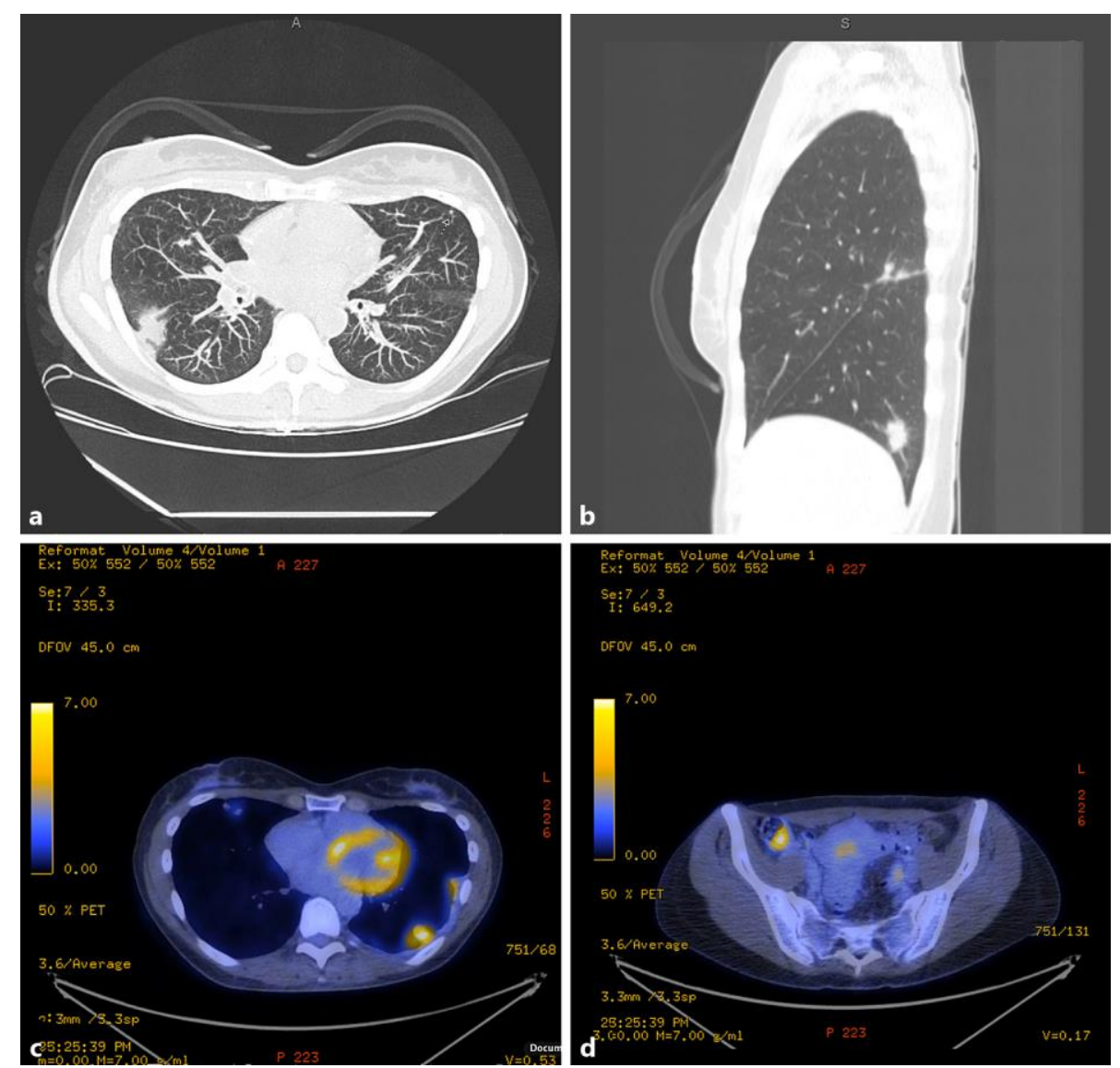

Fig. 1. Axial (a) and sagittal (b) computed tomography images of the chest with multiple pulmonary nodules. c PET scan with fluorodeoxyglucose (FDG)-avid pulmonary nodule in the left lower lobe with associated FDG-avid pleural studding. d FDG-avid mural mass arising from the cecum. 


\section{Case Reports in Gastroenterology} Case Rep Gastroenterol 2016;10:633-639 DOI: $10.1159 / 000452198$ (c) 2016 The Author(s). Published by S. Karger AG, Basel www.karger.com/crg

Chew et al.: A Rare Cause of Pulmonary Nodules
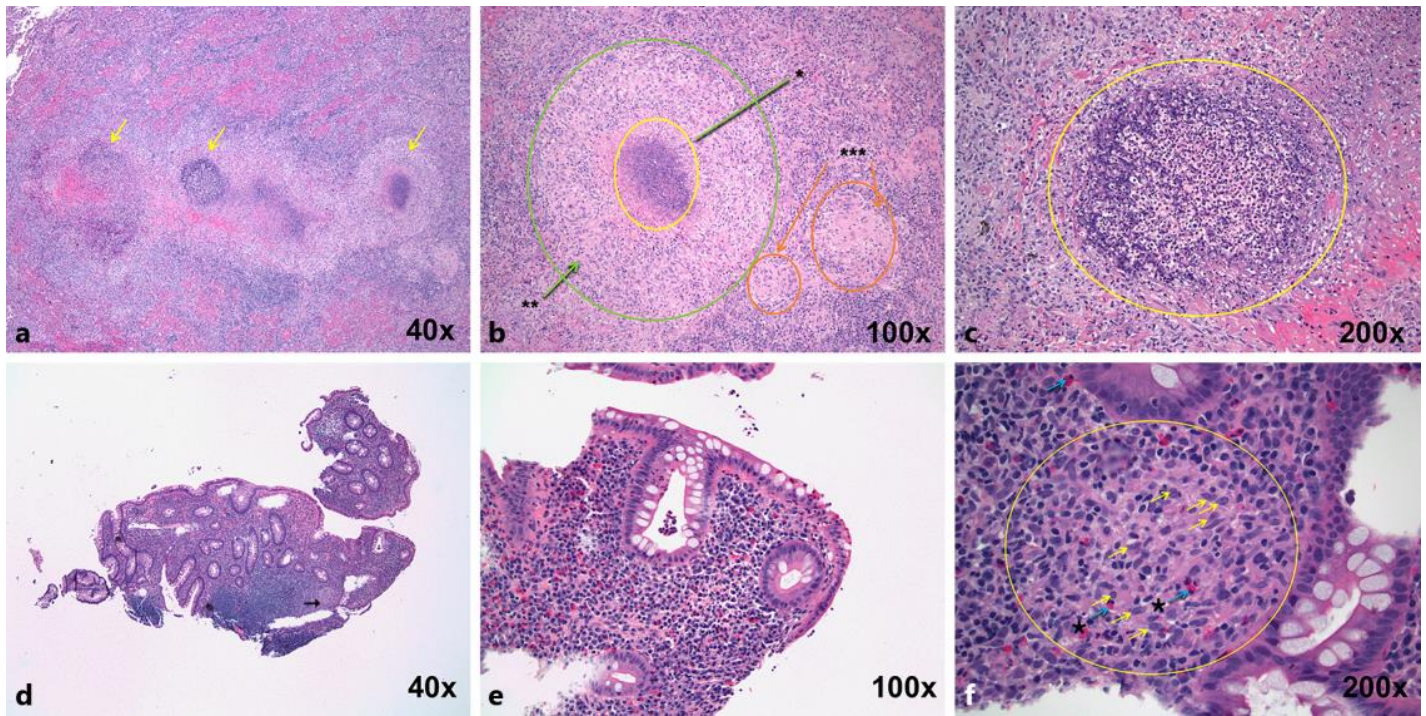

Fig. 2. a Lung biopsy with 3 granulomas (arrows). b Lung biopsy with central necrosis $\left({ }^{*}\right)$, macrophages $\left({ }^{* *}\right)$, and smaller, nonnecrotizing granulomas $\left({ }^{* *}\right)$. c Lung biopsy with suppurative (neutrophilic) central necrosis. d Cecal biopsy under low power (arrow indicates poorly formed granuloma). e Cecal sections with acute cryptitis with crypt abscess. $\mathbf{f}$ Cecal sections under high power with poorly formed granuloma. Arrow, macrophages. * Eosinophils. 